\title{
The Relationship of the Posterior Cranial Fossa with Age and Sex
}

\author{
Relación de la Fosa Craneal Posterior con la Edad y el Sexo
}

\author{
Mahmut Öksüzler'; Sema Polat ${ }^{2}$; Elif Ipek Çay² \& Pinar Göker²
}

ÖKSÜZLER, M.; POLAT, S.; IPEK ÇAY, E. \& GÖKER, P. The relationship of the posterior cranial fossa with age and sex. Int. J. Morphol., 39(5):1371-1382, 2021.

SUMMARY: This paper determined the morphometric measurements of posterior cranial fossa using MRI in Turkish healthy population. Two hundred thirty one $(231 ; 131$ females and 100 males) subjects ranging from 20 up to 85 years were included in this study. Measurements of the posterior cranial fossa were taken from subjects having brain MRI in the Radiology Department, Adana, Turkey. Statistical analysis were done by SPSS 21.00 package programme. ANOVA Test and Chi-Square Test were used to determine the relation between measurements and age groups. The $\mathrm{p}<0.05$ value was considered as significant. The overall means and standard deviations of the measurements were: Clivus length,31.10 $\pm 5.45 \mathrm{~mm}$; McRae line, $32.59 \pm 3.89 \mathrm{~mm}$; Supraocciput length,41.99 $\pm 4.37 \mathrm{~mm}$; Twining line, 79.23 $\pm 5.53 \mathrm{~mm}$; Posterior cranial fossa height, $66.76 \pm 5.06 \mathrm{~mm}$; Cerebellum height, $55.17 \pm 5.29 \mathrm{~mm}$; Clival angle, $125.59^{\circ} \pm 6.57^{\circ}$; Cerebellar tentorium angle, $128.30^{\circ} \pm 7.77^{\circ} \mathrm{mm}$, Occipital protuberance angle, $93.27^{\circ} \pm 8.02^{\circ}$ and hindbrain vertical length, $50.56 \pm 3.47$ mmin females, respectively, whereas the corresponding values were $32.43 \pm 5.99 \mathrm{~mm} ; 32.85 \pm 3.77 \mathrm{~mm} ; 42.46 \pm 4.68 \mathrm{~mm} ; 80.95 \pm 5.94 \mathrm{~mm}$; $69.70 \pm 4.67 \mathrm{~mm} ; 57.01 \pm 3.43 \mathrm{~mm} ; 123.90^{\circ} \pm 7.12^{\circ} 128.80 \pm 8.33^{\circ} ; 95.35 \pm 9.19^{\circ}$ and $52.71 \pm 3.33 \mathrm{~mm}$ in males, respectively. Significant difference was found in some parameters such as twining line, posterior cranial fossa height, cerebellum height and hindbrain vertical length between sex $(\mathrm{p}<0.05)$. Also, ages were divided into six groups as decades. Significant difference between six decades was also found in parameters including McRae line, twining line, posterior cranial fossa height, cerebellum height, clival angle, cerebellar tentorium angle, occipital tuberance angle and hindbrain vertical length $(\mathrm{p}<0.05)$. The posterior cranial fossa dimensions of healthy population provides important and useful knowledge in terms of comparison of abnormalities clinically, and data can be used as an anatomical landmark during surgery involving posterior cranial fossa.

KEY WORDS: Posterior cranial fossa morphometry; Age and sex changes; Clinical importance of angles of posterior cranial fossa.

\section{INTRODUCTION}

Posterior cranial fossa, which accommodates cerebellum, pons, medulla oblangata and brain lobus. It is located at the outlet of the cerebrospinal fluid flow from the ventricular system. The fossa posteriorly is surrounded by the muscles attached to the occipital bone and upper cervical vertebrae (Standring, 2008; Seker \& Rhoton, 2015; Snell, 2015; Arınc1 \& Elhan, 2020). The posterior fossa extends from tentorial incisura to the foramen magnum. It is surrounded by the occipital, temporal, parietal, and sphenoid bones. It is bound in front by the dorsum sellae, the posterior part of the sphenoid body, and the clival part of the occipital bone, os temporale pars mastoidea and pars petrosa facies posterior; os occipitale, and os parietale angulus mastoideus. The brain lobus occipitalis is located at the cerebellar fossa where is at the superior part of the posterior cranial fossa and the two lobus cerebellaris are located at the cerebellar fossa where is the inferior part of the posterior cranial fossa (Standring; Seker \& Rhoton; Snell; Arınc1 \& Elhan). Also, the posterior cranial fossa is the largest and deepest cranial fossa according to anterior and middle cranial fossae, and has the most complicated intracranial anatomy (arterial relationship). The pathways regulating consciousness, vital autonomic functions, and motor activities and sensory reception for the head, body, extremities and the centers for controlling balance and gait (Seker \& Rhoton). Therefore, the fractures of posterior fossa result from hematom which is located at deep back muscles. If the jugular foramen loses the integrity due to trauma, the clinical symtoms emerge due to $9^{\text {th }}, 10^{\text {th }}$ and $11^{\text {th }}$ cranial nerves (Standring; Snell; Arıncı \& Elhan).

\footnotetext{
${ }^{1}$ Adana Medline Hospital, Department of Radiology, Adana, Turkey.

${ }^{2}$ Cukurova University Faculty of Medicine, Department of Anatomy, Adana, Turkey.
} 
Tonsillar herniation is a condition which develops in the pons and cerebellum, and passes from the foramen magnum to cervical vertebral canal (Tastemur et al., 2017; Tsara et al., 2005). Both congenital malformation such as hydrodynamic factors, traction, neuroschisis, and primary paraxial mesodermal failure (embryological defects) and acquired conditions such as pathologies in the circulation of cerebrospinal fluid, intracranial lesions (brain edema, space occupying lesion) or hypotension (chronic cerebro-spinal fluid leak, external lumbar drainage, chronic ventriculoperitoneal shunt) and osseous anomalies of the Posterior Cranial Fossa (PCF) and craniovertebral junction (Paget's disease, craniosynostosis and basilar invagination) and connective tissue disorders, play an important role in tonsillar herniation etiology (Alvarez et al., 1995; Milhorat et al., 1999; Nishikawa et al., 1997; Sekula Jr. et al., 2005; Tastemur et al.; Vurdem et al., 2012).

A reduction in the posterior cranial fossa volume which causes the posterior cranial fossa bones insufficient development to lead to Chiari malformation type I (CMI) formation. This may affect the size of its organs (Badie et al., 1995; Noudel et al., 2009; Tastemur et al.). Because of the structures of the posterior cranial fossa, 10 of the 12 pairs of cranial nerves are located entirely inside the posterior fossa; the ten other pairs have a segment within the posterior fossa (Seker \& Rhoton; Tastemur et al.).

This is the first study evaluating posterior cranial fossa morphometry according to age and sex in only healthy population. Consequently, the dimensions around the posterior cranial fossa have been measured, assessed and evaluated due to complicated and critical importance of the intracranial anatomy and the vital motor and autonomic and sensory functions.

\section{MATERIAL AND METHOD}

This study was carried out from the 231 healthy adult subjects ( 131 females; 100 males) aged 20-85 years over a period of 3 years between January 2019 and 2021. All the test procedures were approved by our University Ethics Committee. Cranial MRI findings were evaluated by radiologist and anatomist (ICC was found as 0.887 ). Healthy adult subjects were selected by criteria of optimal health. Moreover, inclusion criteria for healthy adult subjects were no history of cancer, hemiplegia, intracranial tumoral mass, any psychiatric illness or neurologic disease having surgical operation related brain.

MRI was performed using a $1.5 \mathrm{~T}$ MRI system
(Siemens; Essenza, Erlangen, Germany). Brain MRI protocol including sagittal T2-weighted spin echo (TR:3600, TE: 87 $\mathrm{ms}$; slice thickness $5 \mathrm{~mm}$; gap $1.5 \mathrm{~mm}$ ) was used. The measurements were performed from digital MRI images in a hospital using caliper function with $\mathrm{x} 2$ magnification. Using the midsagittal T2-weighted spin echo image, the following parameters of posterior cranial fossa dimensions were evaluated (Smooker, 1994; Nishikawa et al.; Milhorat et al.; Karagöz et al., 2002; Aydin et al., 2005; Sekula Jr. et al.; Furtado et al., 2009; Dagtekin et al., 2011; Heiss et al., 2012; Hwang et al., 2013; Tastemur et al.; Özalp et al., 2019).

CL. The clivus length: Distance between the basion and the dorsum sellae top edge.

MRL. The McRae line (Foramen magnum anteroposterior length): Distance between basion and the opisthion.

SO. The supraocciput length: Distance between the opisthion and the protuberentia occipitalis interna.

TWL. The twining line (Posterior cranial fossa anteroposterior length): Distance from the dorsum sellae to the internal occipital protuberance.

PCFH. The posterior cranial fossa height: The length of a line perpendicularly drawn from the inferior surface of the splenium corporis callosi to the foramen magnum.

CH. The cerebellum height: The length of the cerebellar hemisphere.

CL. The clival angle (The Welcher basal angle): is formed at the intersection of the nasion-tuberculum line and the tuberculum-basion line. It' average is accepted as $132^{\circ}$ and it should be less than $140^{\circ}$.

CTA. The cerebellar tentorium angle: Angle between the tentorium cerebelli and the supraocciput (tentorium cerebelli slope) or the angle between the supraocciput and the McRae line.

BSVL. The hind brain vertical length: The length of the hind brain midbrain-pons junction and the medullo cervical junction.

OTA.The occipital tuberance angle: The angle between the tentorium and a line connecting the internal occipital protuberance to the opisthion.

The data were divided into two groups: healthy adult female and male subjects (Table I). Furthermore the data were divided also into six groups according to age; subjects aged between 20-30 years for Group 1; 31-40 years for Group 2; 41-50 years for Group 3; 51-60 years for Group 4; and 61-70 years for Group 5; and 71-85 years for Group 6 (Table II).

The measurements were made on the computer screen with an electonic caliper and estimations were expressed as millimeters and degree. The SPSS 21.0 program was used for statistical analysis of the measurement results. From these measurements, means, standard deviations (SD), minimum 
(min.) and maximum (max.) values were calculated; In all statistical analyses; p value under 0.05 was considered statistically significant.

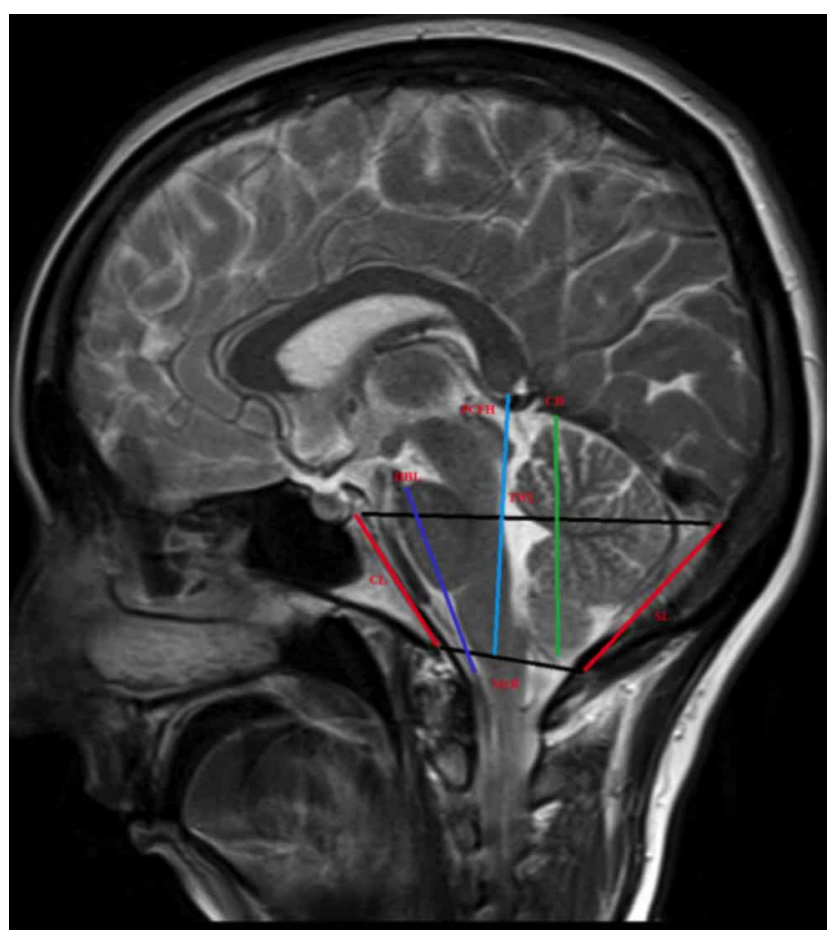

Fig. 1. Posterior cranial fossa linear measurements. CL:Clivus length; MRL: McRae Line; SO:Supraocciput length;TWL:Twining line; $\mathrm{CH}$ :Cerebellar height; PCFH:Posterior cranial fossa height;HBL:Hindbrain length.

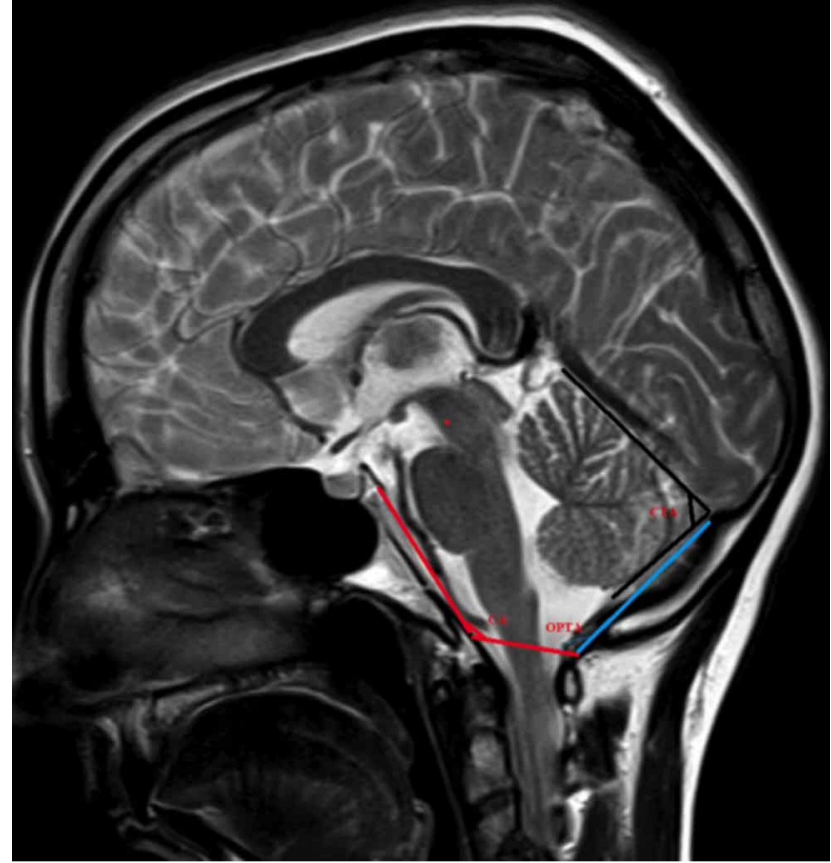

Fig. 2. Posterior cranial fossa angle measurements. CA:Clival angle OTA:Occipital protuberance angle; CTA:Cerebellar tentorium angle.

\section{RESULTS}

The aspect of the posterior cranial fossa in sagittal MR images was shown in Figure 1 and Figure 2.

Table I. Sex related changes of posterior cranial fossa region measurements ( $\mathrm{mm} /$ degree) with magnetic resonance imaging in Turkish healthy population $(\mathrm{n}=231)$.

\begin{tabular}{|c|c|c|c|c|c|c|c|c|}
\hline \multirow[t]{2}{*}{ Posterior Cranial Fossa Values } & \multicolumn{4}{|c|}{ Female (131) } & \multicolumn{4}{|c|}{ Male (100) } \\
\hline & Mean & SD & Min. & Max. & Mean & SD & Min. & Max. \\
\hline Clivus length (mm) & 31.10 & 5.45 & 19.80 & 41.00 & 32.43 & 5.99 & 18.20 & 45.00 \\
\hline P value & \multicolumn{8}{|c|}{0.080} \\
\hline McRae line (mm) & 32.59 & 3.89 & 24.70 & 59.50 & 32.85 & 3.77 & 23.00 & 41.00 \\
\hline P value & \multicolumn{8}{|c|}{0.424} \\
\hline Supraocciput length (mm) & 41.99 & 4.37 & 31.50 & 53.00 & 42.46 & 4.68 & 30.00 & 54.00 \\
\hline Pvalue & \multicolumn{8}{|c|}{0.329} \\
\hline Twining line $(\mathrm{mm})$ & 79.23 & 5.53 & 59.00 & 91.00 & 80.95 & 5.94 & 64.00 & 97.00 \\
\hline$P$ value & \multicolumn{8}{|c|}{0.024} \\
\hline $\begin{array}{l}\text { Posterior Cranial Fossa height } \\
\text { P value }\end{array}$ & \multicolumn{8}{|c|}{$<0.001$} \\
\hline Cerebellum height (mm) & 55.17 & 5.29 & 45.70 & 85.00 & 57.01 & 3.43 & 49.00 & 66.50 \\
\hline P value & \multicolumn{8}{|c|}{0.003} \\
\hline Clival angle $\left(^{\circ}\right)$ & 125.59 & 6.57 & 112.70 & 145.00 & 123.90 & 7.12 & 109.00 & 142.00 \\
\hline P value & \multicolumn{8}{|c|}{0.064} \\
\hline Cerebellar tentorium angle $\left({ }^{\circ}\right)$ & 128.30 & 7.77 & 110.00 & 144.00 & 128.80 & 8.33 & 108.70 & 147.00 \\
\hline$P$ value & \multicolumn{8}{|c|}{0.639} \\
\hline Occipital protuberance angle & 93.27 & 8.02 & 76.00 & 115.10 & 95.35 & 9.19 & 79.00 & 127.10 \\
\hline P value & \multicolumn{8}{|c|}{0.068} \\
\hline Hindbrain vertical length (mm) & 50.56 & 3.47 & 41.60 & 59.00 & 52.71 & 3.33 & 43.00 & 59.00 \\
\hline P value & \multicolumn{8}{|c|}{$<0.001$} \\
\hline
\end{tabular}

*Linear measurements in $\mathrm{mm}$ and angular measurements in degrees. 
The value of minimum, maximum, mean and standard deviation of of the clivus length (CL), the McRae line (MRL), the supraocciput length (SO), the twining line (TWL), posterior cranial fossa height $(\mathrm{PCFH})$, the cerebellum height $(\mathrm{CH})$, clival angle $(\mathrm{CA})$, the cerebellar tentorium angle (CTA), Hindbrain vertical length (HBL), the occipital tuberance angle (OTA) were measured in 231 healthy subjects (131 females and 100 males) were shown in Tables I to IV.
According to Shapiro-Wilk Test, while some measurements including the McRae line, the cerebellum height and the occipital protuberance angle was having no normal distribution $(\mathrm{p}<0.05)$, the clivus length, the supraocciput length, the twinning line, the clival angle, the cranial fossa posterior height, the cerebellar tentorium angle, brain stem vertical length measurements showed normal distribution $(p>0.05)$. For this reason, the parametric tests were performed for the data showing normal distribution,

Table II. The means, standard deviations, and ranges of the posterior cranial fossa region measurements (mm/degree) with magnetic resonance imaging by age groups in Turkish healthy population $(\mathrm{n}=231)$.

\begin{tabular}{|c|c|c|c|c|c|c|}
\hline \multirow{2}{*}{$\begin{array}{l}\text { Posterior Cranial Fossa } \\
\text { Values }\end{array}$} & Decade 1 & Decade 2 & Decade 3 & Decade 4 & Decade 5 & Decade 6 \\
\hline & $\mathrm{n}=65$ & $\mathrm{n}=49$ & $\mathrm{n}=45$ & $\mathrm{n}=22$ & $\mathrm{n}=28$ & $\mathrm{n}=22$ \\
\hline \multirow[t]{2}{*}{ Clivus length $(\mathrm{mm})$} & $31.50 \pm 6.23$ & $31.26 \pm 6.46$ & $32.05 \pm 5.49$ & $31.76 \pm 3.50$ & $32.47 \pm 4.50$ & $31.26 \pm 6.36$ \\
\hline & $20.00-45.00$ & $19.80-44.00$ & $20.50-40.30$ & $27.00-38.00$ & $25.00-43.00$ & $18.20-41.00$ \\
\hline Total mean (min.-max.) & \multicolumn{6}{|c|}{$31.68 \pm 5.71(18.20-45.00)$} \\
\hline$P$ value & \multicolumn{6}{|c|}{0.948} \\
\hline \multirow[t]{2}{*}{ McRae line (mm) } & $34.06 \pm 2.94$ & $32.31 \pm 3.10$ & $31.72 \pm 3.50$ & $33.40 \pm 2.14$ & $31.90 \pm 4.06$ & $31.87 \pm 7.13$ \\
\hline & $27.20-41.00$ & $24.70-41.00$ & $23.00-36.10$ & $29.00-36.00$ & $26.00-40.00$ & $26.00-59.50$ \\
\hline Total mean (min.-max.) & \multicolumn{6}{|c|}{$32.70 \pm 3.83(23.00-59.50)$} \\
\hline $\mathrm{P}$ value & \multicolumn{6}{|c|}{0.011} \\
\hline Supraocciput length (mm) & $43.12 \pm 3.50$ & $40.69 \pm 3.96$ & $42.33 \pm 4.84$ & $42.76 \pm 6.66$ & $41.73 \pm 3.91$ & $42.58 \pm 3.73$ \\
\hline Total mean (min.-max.) & \multicolumn{6}{|c|}{$42.20 \pm 4.37(30.00-54.00)$} \\
\hline$P$ value & \multicolumn{6}{|c|}{0.085} \\
\hline \multirow[t]{2}{*}{ Twining line (mm) } & $81.80 \pm 5.11$ & $80.38 \pm 5.31$ & $78.78 \pm 4.80$ & $80.33 \pm 4.88$ & $79.29 \pm 6.17$ & $76.63 \pm 8.50$ \\
\hline & $70.00-97.00$ & $59.00-89.00$ & $70.70-89.00$ & $74.00-89.00$ & $70.00-91.90$ & $64.00-91.00$ \\
\hline Total mean (min.-max.) & \multicolumn{6}{|c|}{$79.97 \pm 5.76(59.00-97.00)$} \\
\hline $\mathrm{P}$ value & \multicolumn{6}{|c|}{0.005} \\
\hline Posterior Cranial fossa & $70.42 \pm 4.74$ & $67.25 \pm 5.85$ & $67.46 \pm 4.25$ & $68.95 \pm 4.44$ & $66.57 \pm 4.56$ & $64.85 \pm 4.42$ \\
\hline height (mm) & $61.50-83.00$ & $56.00-83.00$ & $57.00-79.00$ & $63.00-76.70$ & $54.10-72.00$ & $58.00-73.30$ \\
\hline Total mean (min.-max.) & \multicolumn{6}{|c|}{$68.03 \pm 5.10(54.10-83.00)$} \\
\hline$P$ value & \multicolumn{6}{|c|}{$<0.001$} \\
\hline \multirow[t]{2}{*}{ Cerebellum height (mm) } & $58.10 \pm 3.81$ & $55.30 \pm 3.49$ & $55.22 \pm 2.57$ & $55.42 \pm 2.18$ & $55.16 \pm 7.40$ & $54.29 \pm 7.53$ \\
\hline & $51.00-66.90$ & $49.00-64.00$ & $50.50-59.00$ & $53.30-60.00$ & $46.20-84.90$ & $45.70-85.00$ \\
\hline Total mean (min.-max.) & \multicolumn{6}{|c|}{$55.97 \pm 4.66(45.70-85.00)$} \\
\hline $\mathrm{P}$ value & \multicolumn{6}{|c|}{0.001} \\
\hline \multirow[t]{2}{*}{ Clival angle $\left({ }^{\circ}\right)$} & $123.34 \pm 6.57$ & $125.46 \pm 6.55$ & $125.87 \pm 8.98$ & $128.44 \pm 3.87$ & $124.00 \pm 5.39$ & $123.45 \pm 6.03$ \\
\hline & $109.20-142.00$ & $115.00-138.00$ & $111.00-145.00$ & $120.00-133.00$ & $109.00-132.60$ & $112.00-132.00$ \\
\hline Total mean (min.-max.) & \multicolumn{6}{|c|}{$124.86 \pm 6.85(109.00-145.00)$} \\
\hline$P$ value & \multicolumn{6}{|c|}{0.034} \\
\hline Cerebellar tentorium angle & $129.79 \pm 5.79$ & $131.27 \pm 6.57$ & $127.43 \pm 10.39$ & $123.02 \pm 6.89$ & $127.85 \pm 7.08$ & $127.21 \pm 10.17$ \\
\hline$\left({ }^{\circ}\right)$ & $115.00-143.20$ & $113.00-147.00$ & $110.00-144.00$ & $113.00-137.00$ & $112.90-142.00$ & $108.70-140.00$ \\
\hline Total mean (min.-max.) & \multicolumn{6}{|c|}{$128.52 \pm 8.01(108.70-147.00)$} \\
\hline $\mathrm{P}$ value & \multicolumn{6}{|c|}{0.001} \\
\hline Occipital & $92.58 \pm 6.66$ & $92.21 \pm 7.51$ & $94.18 \pm 7.86$ & $99.16 \pm 10.86$ & $93.91 \pm 6.63$ & $98.29 \pm 13.66$ \\
\hline protuberance angle $\left(^{\circ}\right)$ & $78.00-107.00$ & $76.60-107.00$ & $80.00-109.50$ & $79.00-113.00$ & $81.00-111.20$ & $76.00-127.10$ \\
\hline Total mean (min.-max.) & \multicolumn{6}{|c|}{$94.15 \pm 8.63(76.00-127.10)$} \\
\hline$P$ value & \multicolumn{6}{|c|}{0.04} \\
\hline Hindbrain vertical length & $52.34 \pm 3.46$ & $51.10 \pm 3.02$ & $50.68 \pm 4.20$ & $52.80 \pm 2.16$ & $51.29 \pm 4.15$ & $50.45 \pm 3.31$ \\
\hline$(\mathrm{mm})$ & $43.00-58.30$ & $44.70-58.00$ & $42.30-59.00$ & $49.00-55.00$ & $41.60-58.00$ & $42.80-59.00$ \\
\hline Total mean (min.-max.) & \multicolumn{6}{|c|}{$51.49 \pm 3.56(41.60-59.00)$} \\
\hline$P$ value & & & & & & \\
\hline
\end{tabular}

*Linear measurements in mm and angular measurements in degrees. 
Table III. The means, standard deviations, and ranges of the posterior cranial fossa region measurements (mm/degree) with magnetic resonance imaging by age groups in Turkish healthy males $(n=100)$.

\begin{tabular}{|c|c|c|c|c|c|c|}
\hline $\begin{array}{l}\text { Posterior Cranial Fossa } \\
\text { Values }\end{array}$ & $\begin{array}{l}\text { Decade } 1 \\
\mathrm{n}=32\end{array}$ & $\begin{array}{l}\text { Decade } 2 \\
\mathrm{n}=15\end{array}$ & $\begin{array}{l}\text { Decade } 3 \\
\mathrm{n}=15\end{array}$ & $\begin{array}{l}\text { Decade } 4 \\
\mathrm{n}=14\end{array}$ & $\begin{array}{l}\text { Decade } 5 \\
\mathrm{n}=15\end{array}$ & $\begin{array}{l}\text { Decade } 6 \\
\mathrm{n}=9\end{array}$ \\
\hline \multirow[t]{2}{*}{ Clivus length (mm) } & $32.43 \pm 7.32$ & $35.51 \pm 6.06$ & $33.37 \pm 3.41$ & $30.34 \pm 2.25$ & $32.45 \pm 5.37$ & $28.94 \pm 7.25$ \\
\hline & $20.00-45.00$ & $23.00-44.00$ & $27.50-39.00$ & $27.00-32.20$ & $25.00-43.00$ & $18.20-38.00$ \\
\hline Total mean (min.-max.) & \multicolumn{6}{|c|}{$32.43 \pm 5.99(18.20-45.00)$} \\
\hline $\mathrm{P}$ value & \multicolumn{6}{|c|}{0.102} \\
\hline \multirow{2}{*}{ McRae line (mm) } & $34.31 \pm 3.60$ & $31.88 \pm 2.52$ & $31.87 \pm 4.14$ & $33.63 \pm 2.26$ & $32.15 \pm 5.05$ & $30.83 \pm 3.66$ \\
\hline & $27.20-41.00$ & $28.00-36.00$ & $23.00-36.10$ & $29.00-36.00$ & $26.00-40.00$ & $26.00-34.70$ \\
\hline Total (min-max.) & \multicolumn{6}{|c|}{$32.85 \pm 3.77(23.00-41.00)$} \\
\hline $\mathrm{P}$ value & \multicolumn{6}{|c|}{0.058} \\
\hline \multirow[t]{2}{*}{ Supraocciput length (mm) } & $43.44 \pm 3.81$ & $41.34 \pm 4.59$ & $42.39 \pm 3.52$ & $42.49 \pm 8.33$ & $41.14 \pm 4.17$ & $43.17 \pm 1.54$ \\
\hline & $34.00-49.00$ & $30.00-48.00$ & $38.00-48.00$ & $31.00-54.00$ & $35.00-47.20$ & $42.00-46.00$ \\
\hline Total mean (min.-max.) & \multicolumn{6}{|c|}{$42.20 \pm 4.37(30.00-54.00)$} \\
\hline $\mathrm{P}$ value & \multicolumn{6}{|c|}{0.608} \\
\hline \multirow[t]{2}{*}{ Twining line $(\mathrm{mm})$} & $82.98 \pm 5.74$ & $82.35 \pm 3.83$ & $78.89 \pm 5.24$ & $80.80 \pm 4.72$ & $81.48 \pm 5.77$ & $74.22 \pm 7.85$ \\
\hline & $73.00-97.00$ & $74.90-89.00$ & $71.00-89.00$ & $75.00-89.00$ & $70.00-91.90$ & $64.00-84.70$ \\
\hline Total mean (min.-max.) & \multicolumn{6}{|c|}{$79.97 \pm 5.76(64.00-97.00)$} \\
\hline $\mathrm{P}$ value & \multicolumn{6}{|c|}{0.002} \\
\hline Posterior cranial fossa & $71.15 \pm 4.72$ & $69.87 \pm 4.92$ & $68.80 \pm 5.46$ & $69.77 \pm 4.94$ & $68.67 \pm 2.66$ & $67.43 \pm 4.45$ \\
\hline height (mm) & $63.90-83.00$ & $63.00-81.00$ & $60.00-79.00$ & $63.00-76.70$ & $63.00-72.00$ & $61.30-73.30$ \\
\hline Total mean (min.-max.) & \multicolumn{6}{|c|}{$69.70 \pm 4.67(60.00-83.00)$} \\
\hline $\mathrm{P}$ value & \multicolumn{6}{|c|}{0.263} \\
\hline \multirow[t]{2}{*}{ Cerebellum height (mm) } & $59.22 \pm 3.65$ & $56.85 \pm 3.19$ & $55.91 \pm 2.43$ & $54.80 \pm 1.54$ & $57.04 \pm 3.41$ & $54.67 \pm 2.31$ \\
\hline & $51.00-66.50$ & $52.00-63.00$ & $52.00-59.00$ & $53.30-57.00$ & $49.00-61.00$ & $51.60-58.20$ \\
\hline Total mean (min.-max.) & \multicolumn{6}{|c|}{$57.01 \pm 3.43(49.00-66.50)$} \\
\hline $\mathrm{P}$ value & \multicolumn{6}{|c|}{$<0.001$} \\
\hline \multirow[t]{2}{*}{ Clival angle $\left({ }^{\circ}\right)$} & $123.13 \pm 7.67$ & $125.33 \pm 7.56$ & $119.20 \pm 5.95$ & $130.83 \pm 1.65$ & $123.08 \pm 5.44$ & $122.77 \pm 6.91$ \\
\hline & $109.20-142.00$ & $115.00-138.00$ & $111.00-145.00$ & $120.00-133.00$ & 109.00-132.60 & $112.00-132.00$ \\
\hline Total mean (min.-max.) & \multicolumn{6}{|c|}{$123.91 \pm 7.12(109.00-142.00)$} \\
\hline $\mathrm{P}$ value & \multicolumn{6}{|c|}{$<0.001$} \\
\hline Cerebellar tentorium angle & $130.67 \pm 6.23$ & $131.24 \pm 7.71$ & $131.03 \pm 8.46$ & $122.46 \pm 8.03$ & $129.46 \pm 6.94$ & $123.18 \pm 12.19$ \\
\hline$\left({ }^{\circ}\right)$ & $117.20-143.20$ & $116.00-147.00$ & $122.00-144.00$ & $113.00-137.00$ & $118.90-142.00$ & $108.70-140.00$ \\
\hline Total mean (min.-max.) & \multicolumn{6}{|c|}{$128.80 \pm 8.33(108.70-147.00)$} \\
\hline$P$ value & \multicolumn{6}{|c|}{0.004} \\
\hline Occipital & $92.82 \pm 6.07$ & $94.74 \pm 6.51$ & $92.73 \pm 9.10$ & $100.54 \pm 12.13$ & $93.98 \pm 6.63$ & $103.91 \pm 13.95$ \\
\hline protuberance angle $\left({ }^{\circ}\right)$ & $78.00-107.00$ & $76.60-107.00$ & $80.00-109.50$ & $79.00-113.00$ & $81.00-111.20$ & $76.00-127.10$ \\
\hline Total mean (min.-max.) & \multicolumn{6}{|c|}{$95.35 \pm 9.19(79.00-127.10)$} \\
\hline$P$ value & \multicolumn{6}{|c|}{0.004} \\
\hline Hindbrain vertical length & $52.39 \pm 4.34$ & $52.49 \pm 2.64$ & $52.84 \pm 3.57$ & $52.97 \pm 2.06$ & $54.03 \pm 2.69$ & $51.32 \pm 1.97$ \\
\hline$(\mathrm{mm})$ & $43.00-58.30$ & $44.90-56.00$ & $48.40-59.00$ & $50.00-55.00$ & $48.60-58.00$ & $49.00-55.00$ \\
\hline Total mean (min.-max.) & \multicolumn{6}{|c|}{$52.71 \pm 3.33(43.00-59.00)$} \\
\hline$P$ value & & & & 505 & & \\
\hline
\end{tabular}

* Linear measurements in mm and angular measurements in degrees.

while non-parametric tests were used for the data not showing normal distribution. Significant difference in some mesurements were found in some parameters such as twinning line, cranial fossa posterior height, cerebellum height and hindbrain vertical length in females and males. Also, the supraoocciput length of females was similar to males.
The clival angle was lower in males than females. Conversely, all measurements except that the SL and CA were higher in males than in females (Table I). Additionally, the distribution of posterior cranial fossa diameters and angles according to age groups of two sexes was shown in Table II. Significant difference between decades was found in parameters including McRae line, twinning line, poste- 
Table IV. The means, standard deviations, and ranges of the posterior cranial fossa region measurements (mm/degree) with magnetic resonance imaging by age groups in Turkish healthy females $(\mathrm{n}=131)$.

\begin{tabular}{|c|c|c|c|c|c|c|}
\hline $\begin{array}{l}\text { Posterior Cranial } \\
\text { Fossa Values }\end{array}$ & $\begin{array}{l}\text { Decade } 1 \\
\mathrm{n}=33\end{array}$ & $\begin{array}{l}\text { Decade } 2 \\
\mathrm{n}=34\end{array}$ & $\begin{array}{l}\text { Decade } 3 \\
\mathrm{n}=30\end{array}$ & $\begin{array}{l}\text { Deacde } 4 \\
\mathrm{n}=8\end{array}$ & $\begin{array}{l}\text { Decade } 5 \\
\mathrm{n}=13\end{array}$ & $\begin{array}{l}\text { Decade } 6 \\
\mathrm{n}=13\end{array}$ \\
\hline $\begin{array}{l}\text { Clivus length } \\
(\mathrm{mm})\end{array}$ & $\begin{array}{l}30.61 \pm 4.90 \\
21.00-40.00\end{array}$ & $\begin{array}{l}29.39 \pm 5.77 \\
19.80-40.80\end{array}$ & $\begin{array}{l}31.40 \pm 6.23 \\
20.50-40.30\end{array}$ & $\begin{array}{l}34.25 \pm 4.03 \\
28.00-38.00\end{array}$ & $\begin{array}{l}32.50 \pm 3.46 \\
27.20-38.00\end{array}$ & $\begin{array}{l}32.86 \pm 5.39 \\
26.00-41.00\end{array}$ \\
\hline $\begin{array}{l}\text { Total mean (min.- } \\
\text { Paxalye }\end{array}$ & \multicolumn{6}{|c|}{$31.10 \pm 5.45(19.80-41.00)$} \\
\hline McRae line (mm) & $\begin{array}{l}33.83 \pm 2.15 \\
29.00-38.00\end{array}$ & $\begin{array}{l}32.50 \pm 3.34 \\
24.70-41.00\end{array}$ & $\begin{array}{l}31.65 \pm 3.21 \\
26.00-36.00\end{array}$ & $\begin{array}{l}33.00 \pm 2.00 \\
31.00-36.00\end{array}$ & $\begin{array}{l}31.60 \pm 2.66 \\
27.40-35.70\end{array}$ & $\begin{array}{l}32.59 \pm 8.88 \\
26.00-59.50\end{array}$ \\
\hline $\begin{array}{l}\text { Total mean (min.- } \\
\text { Phaxlye }\end{array}$ & \multicolumn{6}{|c|}{$32.59 \pm 3.89(24.70-59.50)$} \\
\hline $\begin{array}{l}\text { Supraocciput } \\
\text { length }(\mathrm{mm})\end{array}$ & $\begin{array}{l}42.81 \pm 3.21 \\
38.00-49.00\end{array}$ & $\begin{array}{l}40.41 \pm 3.68 \\
32.00-51.00\end{array}$ & $\begin{array}{l}42.30 \pm 5.43 \\
34.00-53.00\end{array}$ & $\begin{array}{l}43.25 \pm 1.91 \\
41.00-45.00\end{array}$ & $\begin{array}{l}42.40 \pm 3.62 \\
35.70-49.00\end{array}$ & $\begin{array}{l}42.17 \pm 4.72 \\
31.50-49.00\end{array}$ \\
\hline $\begin{array}{l}\text { Total mean (min.- } \\
\text { Pnaxalue }\end{array}$ & \multicolumn{6}{|c|}{$41.99 \pm 4.12(31.50-53.00)$} \\
\hline Twining line $(\mathrm{mm})$ & $\begin{array}{l}80.65 \pm 4.21 \\
70.00-87.00\end{array}$ & $\begin{array}{l}79.51 \pm 5.68 \\
59.00-88.10\end{array}$ & $\begin{array}{l}78.73 \pm 4.66 \\
70.70-88.30\end{array}$ & $\begin{array}{l}79.50 \pm 5.37 \\
74.00-87.00\end{array}$ & $\begin{array}{l}76.77 \pm 5.83 \\
70.10-87.00\end{array}$ & $\begin{array}{l}78.30 \pm 8.84 \\
64.60-91.00\end{array}$ \\
\hline $\begin{array}{l}\text { Total mean (min.- } \\
\text { mav ) } \\
\mathrm{P} \text { value }\end{array}$ & \multicolumn{6}{|c|}{$79.23 \pm 5.53(59.00-91.00)$} \\
\hline $\begin{array}{l}\text { Posterior cranial } \\
\text { fossa height (mm) }\end{array}$ & $\begin{array}{l}69.72 \pm 4.73 \\
61.50-80.90\end{array}$ & $\begin{array}{l}66.09 \pm 5.92 \\
56.00-83.00\end{array}$ & $\begin{array}{l}66.79 \pm 3.40 \\
57.00-72.00\end{array}$ & $\begin{array}{l}67.50 \pm 3.16 \\
64.00-72.00\end{array}$ & $\begin{array}{l}64.15 \pm 5.17 \\
54.10-71.00\end{array}$ & $\begin{array}{l}63.07 \pm 3.56 \\
58.00-68.00\end{array}$ \\
\hline $\begin{array}{l}\text { Total mean (min.- } \\
\text { P value }\end{array}$ & \multicolumn{6}{|c|}{$66.76 \pm 5.06(54.10-83.00)$} \\
\hline $\begin{array}{l}\text { Cerebellum height } \\
(\mathrm{mm})\end{array}$ & $\begin{array}{l}57.01 \pm 3.69 \\
51.00-66.90\end{array}$ & $\begin{array}{l}54.62 \pm 3.43 \\
49.00-64.00\end{array}$ & $\begin{array}{l}54.87 \pm 2.60 \\
50.50-58.00\end{array}$ & $\begin{array}{l}56.50 \pm 2.78 \\
54.00-60.00\end{array}$ & $\begin{array}{l}52.98 \pm 10.00 \\
46.20-84.90\end{array}$ & $\begin{array}{l}54.03 \pm 9.77 \\
45.70-85.00\end{array}$ \\
\hline $\begin{array}{l}\text { Total mean (min.- } \\
\mathrm{P} \text { value }\end{array}$ & \multicolumn{6}{|c|}{$55.17 \pm 5.29(45.70-85.00)$} \\
\hline Clival angle $\left({ }^{\circ}\right)$ & $\begin{array}{l}123.56 \pm 5.42 \\
112.70-138.00\end{array}$ & $\begin{array}{l}125.53 \pm 6.18 \\
115.00-138.00\end{array}$ & $\begin{array}{l}129.20 \pm 8.41 \\
115.50-145.00\end{array}$ & $\begin{array}{l}124.25 \pm 2.87 \\
120.00-127.00\end{array}$ & $\begin{array}{l}125.06 \pm 5.35 \\
118.00-132.60\end{array}$ & $\begin{array}{l}123.92 \pm 5.59 \\
113.00-132.00\end{array}$ \\
\hline $\begin{array}{l}\text { Total mean (min.- } \\
\text { Rasalue }\end{array}$ & & \multicolumn{5}{|c|}{$125.59 \pm 6.57(112.70-145.00)$} \\
\hline $\begin{array}{l}\text { Cerebellar } \\
\text { tentorium angle }\left(^{\circ}\right)\end{array}$ & $\begin{array}{l}128.94 \pm 5.29 \\
115.00-138.00\end{array}$ & $\begin{array}{l}131.28 \pm 6.12 \\
113.00-143.00\end{array}$ & $\begin{array}{l}125.64 \pm 10.92 \\
110.00-144.00\end{array}$ & $\begin{array}{l}124.00 \pm 4.60 \\
120.00-131.00\end{array}$ & $\begin{array}{l}126.00 \pm 7.05 \\
112.90-135.00\end{array}$ & $\begin{array}{l}130.00 \pm 7.84 \\
116.60-140.00\end{array}$ \\
\hline $\begin{array}{l}\text { Total mean (min.- } \\
\text { Praxdlue }\end{array}$ & & \multicolumn{5}{|c|}{$128.30 \pm 7.77(110.00-144.00)$} \\
\hline $\begin{array}{l}\text { Occipital } \\
\text { protuberance }\end{array}$ & $\begin{array}{l}92.34 \pm 7.27 \\
78.00-107.00\end{array}$ & $\begin{array}{l}91.09 \pm 7.73 \\
76.60-107.00\end{array}$ & $\begin{array}{l}94.91 \pm 7.22 \\
80.00-109.50\end{array}$ & $\begin{array}{l}93.83 \pm 6.91 \\
79.00-113.00\end{array}$ & $\begin{array}{l}94.40 \pm 12.52 \\
81.00-111.20\end{array}$ & $\begin{array}{l}94.40 \pm 12.51 \\
76.00-127.10\end{array}$ \\
\hline $\begin{array}{l}\text { Total mean (min.- } \\
\text { Raxalue }\end{array}$ & \multicolumn{6}{|c|}{ 93.23. $\pm 8.09(76.00-115.10)$} \\
\hline $\begin{array}{l}\text { Hindbrain vertical } \\
\text { length }(\mathrm{mm})\end{array}$ & $\begin{array}{l}52.31 \pm 2.38 \\
46.60-56.00\end{array}$ & $\begin{array}{l}50.49 \pm 3.00 \\
44.70-58.00\end{array}$ & $\begin{array}{l}49.59 \pm 4.12 \\
42.30-59.00\end{array}$ & $\begin{array}{l}52.50 \pm 2.45 \\
49.00-55.00\end{array}$ & $\begin{array}{l}48.12 \pm 3.18 \\
41.6-53.00\end{array}$ & $\begin{array}{l}49.85 \pm 3.95 \\
42.80-55.00\end{array}$ \\
\hline $\begin{array}{l}\text { Total mean (min.- } \\
\text { Pnaxilde }\end{array}$ & \multicolumn{6}{|c|}{$50.56 \pm 3.47(41.60-59.00)$} \\
\hline
\end{tabular}

*Linear measurements in mm and angular measurements in degrees..

rior cranial fossa height, cerebellum height, clival angle, cerebellar tentorium angle, occipitotuberance angle and hindbrain vertical length $(\mathrm{p}<0.05)$. The highest value was obtained in decades 5 in clivus length, decade 1 in McRae line, supraoociput length, twinning line, posterior cranial fossa, cerebellum height, decade 4 in clival angle, occipital protuberance angle and hindbrain vertical length; in decade 2 in cerebellar tentorium angle in healthy population. Also, the lowest value was in decade 2 in the clivus length, supraoociput length and occipital protuberance angle; in decade 3 in McRae line; in decade 6 in twinning line, posterior cranial fossa length, the cerebellum length and hindbrain vertical length; in decade 1 in clival angle; and in decade 4 in cerebellar tentorium angle in healthy population (Table II). 
In males, the highest value was obtained in decade 2 in clivus length and cerebellar tentorium angle; decade 1 in McRae line, Supraoociput length, twinning line, posterior cranial fossa, cerebellum height, decade 4 in clival angle; decade 6 in occipital protuberance angle and decade 5 in hindbrain vertical length. The lowest value was in decade 6 in clivus length, McRae line, twinning line, posterior cranial fossa, cerebellum height and hindbrain vertical length; decade 5 in supraocciput length; decade 3 in clival angle and occipital protuberance angle; decade 4 in cerebellar tentorium angle in males (Table III).

In females, the highest value was obtained in decade 4 in clivus length, Supraoociput length and hindbrain vertical length; decade 1 in McRae line, twinning line, posterior cranial fossa, cerebellum height, decade 3 in clival angle and occipital protuberance angle and decade 2 in cerebellar tentorium angle. The lowest value was in decade 2 in clivus length, supraocciput length, occipital protuberance angle; decade 5 in McRae line, twinning line, cerebellum height and hindbrain vertical length; decade 6 in posterior cranial fossa height; decade 1 in clival angle and decade 4 in cerebellar tentorium angle in females (Table IV). Additionally, the Post Hoc test results of healthy males and females were shown in Tables V and VII.

\section{DISCUSSION}

The supraocciput length of healthy females was similar to healthy males and the clival angle was higher in healthy females than healthy males. The other post cranial measurements was lower in females than males. In the literature to date, no studies were found in which posterior cranial fossa measurements were performed with only healthy subjects and age groups. It was observed that the studies carried out are generally related to Arnold Chiari Malformation. For this reason, we can say that our study is the first on age and sex comparisons of posterior cranial fossa.

In literature, the tonsillar herniation was defined as supraocciput length. The studies reported that the supraocciput length was lower in CMI patients than healthy subjects (Sekula Jr. et al.). There was significant difference in some studies (Milhorat et al., 99; Karagöz et al.; Aydin et al.), while some studies showed no significant difference (Sekula Jr. et al.; Furtado et al.; Hwang et al.). Clinical criteria for tonsillar descent was accepted as $3-5 \mathrm{~mm}$ or more below the foramen magnum and compatible symptomatology (Sekula Jr. et al.). The supraocciput length (tonsillar herniation) was found as 40.50 \pm 5.9 $\mathrm{mm}$ and $41.80 \pm 6.2 \mathrm{~mm}$ in CMI patients aged between 17-65 years (mean 48 years) and control group aged between 34-68 years (mean 51 years ) in USA, respectively (Sekula Jr. et al.). Milhorat et al. study performed by patients with $50 \mathrm{CMI}$ and 50

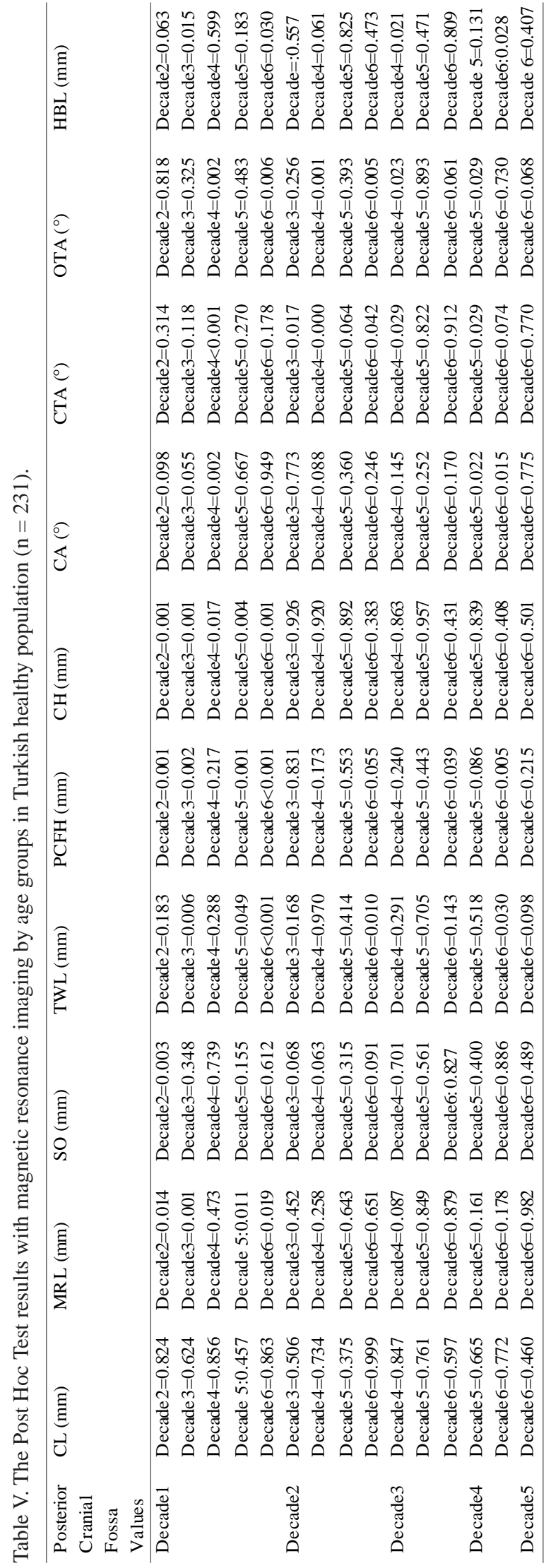


ÖKSÜZLER, M.; POLAT, S.; IPEK ÇAY, E. \& GÖKER, P. The relationship of the posterior cranial fossa with age and sex. Int. J. Morphol., 39(5):1371-1382, 2021.

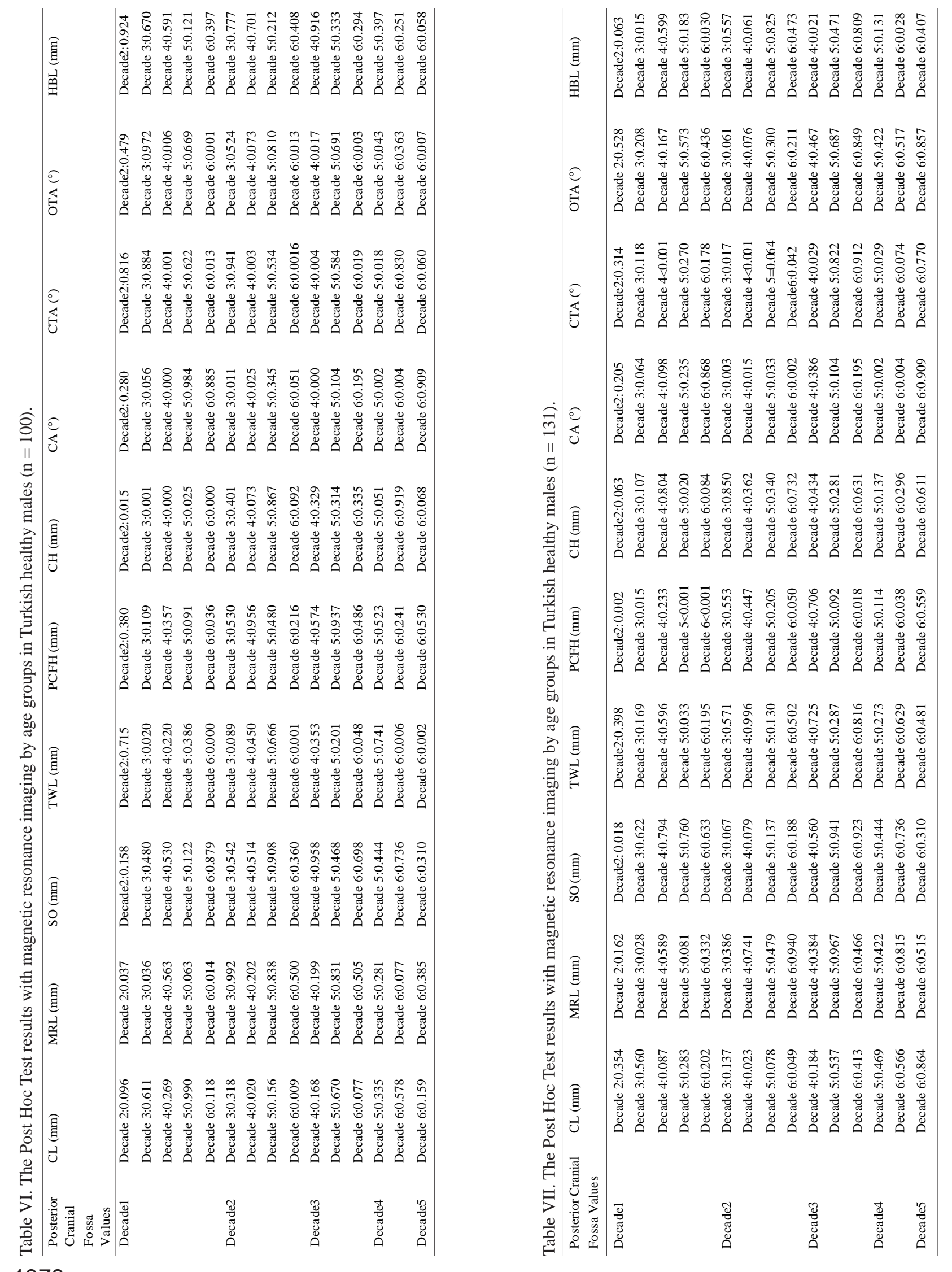


healthy subjects evaluated the supraocciput length as $37.7 \pm 5.9 \mathrm{~mm}$ and $41.8 \pm 5.2 \mathrm{~mm}$, respectively. In a study performed with $22 \mathrm{CMI}$ patients and 21 healthy subjects by Karagöz et al., the corresponding value was $38 \pm 5.2 \mathrm{~mm}$ and $40.9 \pm 7 \mathrm{~mm}$, respectively (Karagöz et al.).

The mean length of supraocciput length in the the 30 Japanese Chiari Malformation Type I $(38.9 \mathrm{~mm})$ was not significantly lower than that in the 50 Japanese control group (48.1 mm) (Nishikawa et al.).

The mean value of the supraocciput length was found as $38 \mathrm{~mm}$ (range $32-45 \mathrm{~mm}$ ) in Indian patients with CMI, while the same value was $42 \mathrm{~mm}$ (range $35-63 \mathrm{~mm}$ ) in Indian healthy subjects (Furtado et al.). In a study of Aydin et al's performed with 30 CMI patients aged between $35.1 \pm 12.7$ years and 60 healthy subjects aged between $52.2 \pm 18.2$ years, the corresponding value was found $42.1 \pm 9.3 \mathrm{~mm}$ and $46.7 \pm 4.3 \mathrm{~mm}$, respectively (Karagöz et al.). In Korean population the same measurement was determined as 34.29 $\mathrm{mm}$ in 12 patients with CMI and $40.18 \mathrm{~mm}$ in 24 healthy subjects (Hwang et al.). In USA population, Heiss et al. study reported that the same measurement was found as $40.1 \pm$ $4.0 \mathrm{~mm}$ in $48 \mathrm{CMI}$ patients and $41.5 \pm 4.4 \mathrm{~mm}$ in 18 healthy subjects, respectively. In this paper the corresponding value was $42.46 \pm 4.68 \mathrm{~mm}$ and $41.99 \pm 4.12 \mathrm{~mm}$ in males and females. Due to these data, we found differences in the mean values of supraocciput length or tonsillar herniation of studies with our population. According to this data, Karagöz et al. values were found greater than ours. However, Indians' values were similar to our data.

Clivus plays a critical role in both the skull base anatomic growth and congenial anomalies detection. Also, the clival angle which the angulation made with the skull base, has a significant role in terms of anterior endoscopic approaches, and congenital malformation diagnosis and fetal period evaluations. Clivus length, clivus shape and clival angle may be effective on clinical diseases which contains coloboma, heart defects, atresia choanae (also known as choanal atresia), growth retardation, genital abnormalities, and ear abnormalities, and basilar invagination, Arnold Chiari Malformation and platybasia. Considering that clivus anomalies are associated with diseases such as platybasia, basilar invagination, CHARGE syndrome or Chiari Type I, the data of the present study can be used for the detection of clivus anomalies as well as choosing the type of approach to the skull base (Özalp et al.).

Tastemur et al., reported the clivus length as $37.46 \pm 3.75 \mathrm{~mm}$ in the CMI patients and $39.78 \pm 4.62 \mathrm{~mm}$ in control group. Aydin et al. performed with 60 patients having Chiari Type I Malformation mean aged $35.1 \pm 12.70$ years and 30 healthy subjects mean aged $52.2 \pm 18.2$ years that the clivus length was $39.0 \pm 7.7 \mathrm{~mm}$ and $48.4 \pm 4.9 \mathrm{~mm}$, respectively. In Turkish population the clivus length was measured as $39.7 \mathrm{~mm}$ (anatomical) and $39.4 \mathrm{~mm}$ (radiological) in dry skull. Also, the same value was reported as $40.8 \mathrm{~mm}$ in CMI patients and $42.4 \mathrm{~mm}$ in healthy subjects in CT examination (Dagtekin et al., 2011). The mean value of the clivus length was found as $35 \mathrm{~mm}$ in Indian patients with CMI, while the same value was $3.8 \mathrm{~mm}$ in Indian healthy subjects. Also, this parameter was $35 \mathrm{~mm}$ and 43.9 $\mathrm{mm}$ in patients with pediatric CMI and adult CMI (Furtado et al.). The clivus length was found as $32.95 \pm 8.7 \mathrm{~mm}$ and $43.00 \pm 6.6 \mathrm{~mm}$ in CMI patients aged between $17-65$ years (mean 48 years) and control group aged between $34-68$ years (mean 51 years) in USA, respectively (Sekula Jr. et al.). The mean length of the clivus in the 30 Japanese Chiari Malformation Type I $(49.7 \mathrm{~mm})$ was not significantly lower than in the 50 Japanese control group $(50.1 \mathrm{~mm})$ (Nishikawa et al.). Milhorat et al. study performed by patients with 50 $\mathrm{CMI}$ and 50 healthy subjects evaluated the clivus length as $40.4 \pm 5.1 \mathrm{~mm}$ and $36.6 \pm 4.2 \mathrm{~mm}$, respectively (Milhorat $e t$ al.). In a study performed with 22 CMI patients and 21 healthy subjects by Karagöz et al., the corresponding value was $35.5 \pm 6.7 \mathrm{~mm}$ and $40.4 \pm 4.1 \mathrm{~mm}$, respectively. In Korean population the same measurement was significantly lower in the CMI patients (mean, 22.81) than in the control group (mean, $39.84 \mathrm{~mm})(\mathrm{p}<0.001)$ (Hwang et al.). In USA population, Heiss et al. study reported that the same measurement was found as $38.6 \pm 3.4 \mathrm{~mm}$ in $48 \mathrm{CMI}$ patients and $43.2 \pm 3.5 \mathrm{~mm}$ in 18 healthy subjects, respectively.

Welcher or basilar angle is defined as clival angle. The angle's average is accepted as $132^{\circ}$ and it should be less than $140^{\circ}$. It increases when the skull base is abnormally flattened (Smooker; Özalp et al.) It would seem that when the literature is scanned for the angle in question, it is also defined as below 140 degrees in subjects without malformation.This angle plays an important role in clinical disease such as CHARGE syndrome or platybasia in terms of diagnostic criterion. In CHARGE syndrome, the angle was higher than normal (Smooker; Özalp et al.). The clival angle was reported as $126.12 \pm 9.51^{\circ}$ (with caliper) and $124.37 \pm 10.86^{\circ}$ (with Computed Tomography) in dry skull (Özalp et al.). Additionally, the clival angle which was defined as the angle between the clivus and the McRae line $(\mathrm{p}<0.024)$ was reported as $125.42 \mathrm{~mm}$ in patients having CMI and $117.42 \mathrm{~mm}$ in healthy subjects (Hwang et al.). In this study, the correponding value was $125.59^{\circ} \pm 6.57^{\circ}$ and $123.90^{\circ} \pm 7.12^{\circ}$ in females and males.

The narrowness of hind brain by underdevelopment PCF, also in bone structure may be the primary reason of CMI and syringomyelia (Aydin et al., 2005; Tastemur et al.). 
The underdevelopment in the intrauterine life of bony structures leads to downward herniation of the PCF contents as the caudal hindbrain develops normally (Nishikawa et al.; Aydin et al). A less developed occipital bone as is in the CMI, may occur with the reason of underdevelopment of the occipital somite originating from the paraxial mesoderm. This induces overcrowding in the posterior cranial fossa. It has the normally developed hindbrain. It secondarily induces a downward herniation of the brain as well as an upward shift of the cerebellar tentorium. Basilar invagination is characterized with more severe downward herniation of the hindbrain because of the more severely underdeveloped occipital enchondrium. It induces the overcrowding of the posterior cranial fossa (Nishikawa et al.). In Turkish CMI and healthy subjects the posterior fossa height was reported between $57.31 \pm 4.21 \mathrm{~mm}$ and $124.7 \pm 15.7 \mathrm{~mm} ; 60.54 \pm 4.30$ $\mathrm{mm}$ and $141.2 \pm 6.8 \mathrm{~mm}$, respectively. Also, the height of the posterior fossa is statistically significantly lower in the CMI patients compared to the control subjects (Nishikawa et al.; Tastemur et al.). In this paper, the corresponding value was $66.76 \pm 5.06 \mathrm{~mm}$ and $69.70 \pm 4.67 \mathrm{~mm}$ in healthy females and males, respectively. The highest value was obtained between 20-30 years, while the lowest was 70 and over.

McRae line is defined as a point between basion which is the anterior middle part of the foramen magnum and opisthion which is the posterior middle of foramen magnum and marks the anteroposterior length of foramen magnum. The foramen magnum located in the middle of the posterior cranial fossa, is the major hollow. In literarure, foramen magnum AP length measurement was contradictory. Some studies found high the same value in patients with CMI (Karagöz et al.; Aydin et al., 2005; Sekula Jr. et al.; Dagtekin et al.; Snell), while some studies found the foramen magnum AP length lower in CMI patients than healthy subjects (Hwang et al.). Aydin et al., performed with 60 patients having Chiari Type I Malformation mean aged $35.1 \pm 12.70$ years and 30 healthy subjects mean aged $52.2 \pm 18.2$ years that the foramen magnum AP length was $31.7 \pm 6.1 \mathrm{~mm}$ and $25.2 \pm 3.8 \mathrm{~mm}$, respectively. The same value was measured as $34.5 \mathrm{~mm}$ (anatomical) and $34.3 \mathrm{~mm}$ (radiological) in dry skull. Also, the same value was reported as $40.1 \mathrm{~mm}$ in CMI patients and $32.4 \mathrm{~mm}$ in healthy subjects in CT examination (Dagtekin et al.). In a comparison study performed with 22 Chiari Malformation Type I patients and 21 normal population by Karagöz et al., the McRae line was measured as $35.3 \pm 4.8 \mathrm{~mm}$ and $34.3 \pm 3.9 \mathrm{~mm}$ in CMI patients and healthy Turkish population. In Korean population, the same measurement was determined as 24.78 $\mathrm{mm}$ in 12 patients with CMI and $29.54 \mathrm{~mm}$ in 24 healthy subjects (Hwang et al.). The AP length of foramen magnum was found as $43.55 \pm 4.9 \mathrm{~mm}$ and $42.52 \pm 5.9 \mathrm{~mm}$ in CMI patients aged between 17-65 years (mean 48 years) and control group aged between 34-68 years (mean 51 years ) in USA, respectively (Sekula Jr. et al.).

In Turkish CMI and healthy subjects the posterior fossa height was reported between $35.43 \pm 3.34 \mathrm{~mm}$ and $33.74 \pm 3.57 \mathrm{~mm}$, respectively. Also, the measurement was statistically significantly higher in the CMI patients compared to the control subjects (Tastemur et al.). The twinning line or posterior cranial fossa AP length may be defined as distance between the dorsum sellae and the protuberentia occipitalis interna (Tastemur et al.). The increase in the anteroposterior dimension of the posterior fossa was attributed to a compensatory remodeling of a small posterior fossa. In a study performed with $22 \mathrm{CMI}$ patients and 21 healthy subjects by Karagöz et al. the corresponding value was $38 \pm 5.2 \mathrm{~mm}$ and $93.7 \pm 3.6 \mathrm{~mm}$, respectively. In a study of Aydin et al's performed with $30 \mathrm{CMI}$ patients aged between $35.1 \pm 12.7$ years and 60 healthy subjects aged between $52.2 \pm 18.2$ years, the corresponding value was found $60.4 \pm 10.6 \mathrm{~mm}$ and $74.7 \pm 3.5 \mathrm{~mm}$, respectively (Aydin et al.). The twining line was found as $84.55 \pm 7.8 \mathrm{~mm}$ and $87.32 \pm 6.6 \mathrm{~mm}$ in CMI patients aged between $17-65$ years (mean 48 years) and control group aged between 34-68 years (mean 51 years) in USA, respectively (Sekula Jr. et al.). Additionally, in Korean population the hindbrain vertical length was determined as $52.99 \mathrm{~mm}$ in 12 patients with CMI and $51.18 \mathrm{~mm}$ in 24 healthy subjects (Hwang et al.). The corresponding value was measured as $50.56 \mathrm{~mm}$ and $52.71 \mathrm{~mm}$ in healthy females and males in the present study. The same value was found as $42.9 \mathrm{~mm}$ in CMI patients and $44.6 \mathrm{~mm}$ in healthy subjects, respectively.

Tastemur et al. study showed that the size of the cerebellum was greater in the CMI patients than normal subjects. For this reason, the risk of tonsillar herniation may be higher than the normal population in patients with a small posterior cranial fossa and a large cerebellum. The significant difference was found in the superior-inferior cerebellar hemisphere measurement between patients having CMI and healthy subjects in Tastemur et al. and Hwang et al. studies. The other study performed by Nishikawa et al. and Sekula Jr. et al., reported that there was no significant difference. In Turkish population, the superior-inferior cerebellar hemisphere was found as $59.13 \pm 5.33 \mathrm{~mm}$ in the CMI patients and $53.32 \pm 5.37 \mathrm{~mm}$ in the healthy subjects (Tastemur et al.). The corresponding value was measured as $47.36 \pm 7.9 \mathrm{~mm}$ (patients with CMI) and $47.04 \pm 4.8 \mathrm{~mm}$ (healthy subjects) (Sekula Jr. et al.); $49.1 \mathrm{~mm}$ in the Chiari group and $47.7 \mathrm{~mm}$ in the healthy subjects (Nishikawa $e t$ al.); and $58.50 \mathrm{~mm}$ and $53.89 \mathrm{~mm}$ (patients with CMI and healthy subjects) (Hwang et al.). In this paper, the same value was $55.17 \pm 5.29 \mathrm{~mm}$ and $57.01 \pm 3.43 \mathrm{~mm}$ in healthy females and males. 
Angle measurements obtained in morphometric studies have supported the view that the tentorium was pushed forward and became steeper to compensate the undersized bony posterior fossa (Karagöz et al.). The angle between the tentorium and a line connecting the internal occipital protuberance to the opisthion was used to estimate the steepness of the cerebellar tentorium. In Karagöz et al. study, the angle between tentorium and opisthion was $91 \pm 21^{\circ}$ and $85 \pm 6^{\circ}$ in patients with CMI and normal subjects, respectively. In a study performed by Tastemur et al, in Turkish population, the tentorial angle was found as $89.08 \pm 7.78^{\circ}$ in the CMI patients and $90.33 \pm 7.66^{\circ}$ in the controls. Also, the same value was reported as $86.1^{\circ}$ in CMI patients and $84.4^{\circ}$ in healthy subjects in CT examination (Dagtekin et al.). Milhorat et al's study performed by patients with $50 \mathrm{CMI}$ and 50 healthy subjects evaluated the supraocciput length as $90.0 \pm 7.7^{\circ}$ and $82.5 \pm 7.2^{\circ} \mathrm{mm}$, respectively (Milhorat et al.). In Korean population the same measurement was determined as $116.83^{\circ}$ in 12 patients with CMI and $103.78^{\circ}$ in 24 healthy subjects (Hwang et al.). The tentorial angle was found as $41.27 \pm 6.5^{\circ}$ and $34.84 \pm 7.2^{\circ}$ in CMI patients aged between 17-65 years (mean 48 years) and control group aged between 34-68 years (mean 51 years) in USA, respectively (Sekula Jr. et al.). The mean of the corresponding value in the the 30 Japanese Chiari Malformation Type I $\left(44.6^{\circ}\right)$ was not significantly lower than that in the 50 Japanese control group $\left(36.2^{\circ}\right.$ ) (Nishikawa et al.). In Korean population the same measurement was determined as 130.91 degree in 12 patients with CMI and 123.08 degree in 24 healthy subjects (Hwang et al.). In our study, the angle was measured as $128.30^{\circ} \pm 7.77$ in healthy females and $128.80^{\circ} \pm 8.33$ in healthy males.

The clival angle plays an important role in estimating the steepness of clivus as well as the occipital protuberance angle. There were some differences in measurements. We consider that these differences in all data could be a result of such factors such as race, genetic variables, demographic variables (age, sex), many diseases and the measurement method differences in posterior cranial fossa measurements.

\section{CONCLUSIONS}

The findings of various studies have been compared with those in the current study and demonstrate differences and similarities in the distances and angles measured. presenting morphometric and angle data play an important role in surgical procedures in the region and viewing the skull base and images of the posterior cranial fossa due to the vulnerability in surgical operation. Having a detailed knowledge of the anatomical structures of this region can help to prevent intraoperative damage to neurovascular structures, as well as improve surgical techniques and decrease the morbidity and mortality rate during the surgical procedures.

While many studies have been published based on posterior cranial fossa, it is concluded that the observations of the past studies were related to especially Chiari Malformation type I. In this regard, we can say that this is the first study considering posterior cranial fossa morphometry according to especially, age related changes and sex differences in Turkish healthy population. Also, our study provides important information and reference data related to posterior cranial fossa measurements in terms of clinical work. These presented findings, which include the age and sex related normal values changes, will provide an evaluation opportunity to data regarding age and sex related posterior cranial fossa studies and shed light on patients with CHARGE disorders or CMI diseases or platybasia and in the aging process.

ÖKSÜZLER, M.; POLAT, S.; IPEK ÇAY, E. \& GÖKER, P. Relación de la fosa craneal posterior con la edad y el sexo. Int. J. Morphol., 39(5):1371-1382, 2021.

RESUMEN: Se determinaron las medidas morfométricas de la fosa craneal posterior mediante resonancia magnética en una población turca. Se incluyeron doscientos treinta y un $(231 ; 131$ mujeres y 100 hombres) sujetos sanos con edades entre los 20 y los 85 años. Por medio de una resonancia magnética cerebral se tomaron medidas de la fosa craneal posterior de sujetos en el Departamento de Radiología de Adana, Turquía. El análisis estadístico se realizó mediante el programa de SPSS 21.00. Se utilizó la prueba ANOVA y la prueba de chi-cuadrado para determinar la relación entre las medidas y los grupos de edad. Se consideró significativo el valor de $\mathrm{p}<0,05$. Las medias generales y las desviaciones estándar de las medidas en las mujeres fueron: longitud del

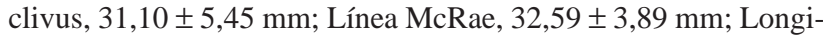
tud del supraoccipucio, 41,99 \pm 4,37 mm; línea de Twining (desde el tubérculo selar hasta la confluencia de los senos), 79,23 $\pm 5,53$ $\mathrm{mm}$; Altura posterior de la fosa craneal, $66,76 \pm 5,06 \mathrm{~mm}$; Altura del cerebelo, $55,17 \pm 5,29 \mathrm{~mm}$; Ángulo clival, $125,59^{\circ} \pm 6,57^{\circ}$; Ángulo del tentorio cerebeloso, $128,30^{\circ} \pm 7,77^{\circ} \mathrm{mm}$, Angulo de protuberancia occipital, $93,27^{\circ} \pm 8,02^{\circ}$ y Longitud vertical del rombencéfalo, $50,56 \pm 3,47 \mathrm{~mm}$. En los hombres los valores obtenidos fueron $32,43 \pm 5,99 \mathrm{~mm} ; 32,85 \pm 3,77 \mathrm{~mm}$; 42,46 $\pm 4,68$ $\mathrm{mm} ; 80,95 \pm 5,94 \mathrm{~mm} ; 69,70 \pm 4,67 \mathrm{~mm} ; 57,01 \pm 3,43 \mathrm{~mm} ; 123,90$ $\circ \pm 7,12^{\circ} 128,80 \pm 8,33^{\circ} ; 95,35 \pm 9,19^{\circ}$ y $52,71 \pm 3,33 \mathrm{~mm}$, respectivamente. Se encontraron diferencias significativas entre ambos sexos $(\mathrm{p}<0,05)$ en algunos de los parámetros, como la línea de Twining, la altura de la fosa craneal posterior, la altura del cerebelo y la longitud vertical del rombencéfalo. También se encontró una diferencia significativa entre las edades de los individuos (división en seis décadas) en los parámetros que incluyen la línea de McRae, la línea de Twining, la altura de la fosa craneal posterior, 
la altura del cerebelo, el ángulo de clivación, el ángulo de la tienda del cerebelo, el ángulo de protuberancia occipital y la longitud vertical del rombencéfalo ( $\mathrm{p}<0,05$ ). La población estudiada nos proporciona información importante y útil en términos de comparación clínica de anomalías y los datos pueden eventualmente ser utilizados como un punto de referencia anatómico durante la cirugía que involucra la fosa craneal posterior.

PALABRAS CLAVE: Morfometría de la fosa craneal posterior; Cambios de edad y sexo; Importancia clínica de los ángulos de la fosa craneal posterior.

\section{REFERENCES}

Alvarez, D.; Requena, I.; Arias, M.; Valdés, L.; Pereiro, I. \& De la Torre, R. Acute respiratory failure as the first sign of Arnold-Chiari malformation associated with syringomyelia. Eur. Respir. J., 8(4):6613, 1995.

Arıncı, K. \& Elhan, A. Anatomi 1.-2. Cilt, 7. Baskı, Ankara, Günes Tıp Kitabevleri, 2020

Aydin, S.; Hanimoglu, H.; Tanriverdi, T.; Yentur, E. \& Kaynar, M. Y. Chiari type I malformations in adults: a morphometric analysis of the posterior cranial fossa. Surg. Neurol., 64(3):237-41, 2005.

Badie, B.; Mendoza, D. \& Batzdorf, U. Posterior fossa volume and response to suboccipital decompression in patients with Chiari I malformation. Neurosurgery, 37(2):214-8, 1995.

Dagtekin, A.; Avci, E.; Kara, E.; Uzmansel, D.; Dagtekin, O.; Koseoglu, A.; Talas, D. \& Bagdatoglu, C. Posterior cranial fossa morphometry in symptomatic adult Chiari I malformation patients: comparative clinical and anatomical study. Clin. Neurol. Neurosurg., 113(5):399403, 2011.

Furtado, S. V.; Reddy, K. \& Hegde, A. S. Posterior fossa morphometry in symptomatic pediatric and adult Chiari I malformation. J. Clin. Neurosci., 16(11):1449-54, 2009.

Heiss, J. D.; Suffredini, G.; Bakhtian, K. D.; Sarntinoranont, M. \& Oldfield, E. H. Normalization of hindbrain morphology after decompression of Chiari malformation Type I. J. Neurosurg., 117(5):942-6, 2012.

Hwang, H. S.; Moon, J. G.; Kim, C. H.; Oh, S. M.; Song, J. H. \& Jeong, J. H. The comparative morphometric study of the posterior cranial fossa : what is effective approaches to the treatment of Chiari malformation type 1? J. Korean Neurosurg. Soc., 54(5):405-10, 2013.

Karagöz, F.; Izgi, N. \& Sencer, S. K. Morphometric measurements of the cranium in patients with Chiari type I malformation and comparison with the normal population. Acta Neurochir. (Wien), 144(2):165-71, 2002.

Milhorat, T. H.; Chou, M. W.; Trinidad, E. M.; Kula, R. W.; Mandell, M.; Wolpert, C. \& Speer, M. C. Chiari I malformation redefined: clinical and radiographic findings for 364 symptomatic patients. Neurosurgery, 44(5):1005-17, 1999.

Nishikawa, M.; Sakamoto, H.; Hakuba, A.; Nakanishi, N. \& Inoue, Y. Pathogenesis of Chiari malformation: a morphometric study of the posterior cranial fossa. J. Neurosurg., 86(1):40-7, 1997.

Noudel, R.; Jovenin, N.; Eap, C.; Scherpereel, B.; Pierot, L. \& Rousseaux, P. Incidence of basioccipital hypoplasia in Chiari malformation type I: comparative morphometric study of the posterior cranial fossa. Clinical article. J. Neurosurg., 111(5):1046-52, 2009.

Özalp, H.; Beger, O.; Erdogan, O.; Kara, E.; Hamzaoglu, V.; Vayisog `lu, Y.; Dagtekin, A.; Öztürk, A. H.; Bagdatoglu, C. \& Talas, D. Ü. Morphometric Analysis of the Clivus in Human Dry Skulls: A Radioanatomical Study. Ankara Üniversitesi Tıp Fakültesi Mecmuası, 72(2):227-30, 2019.
Sekula Jr., R. F.; Jannetta, P. J.; Casey, K. F.; Marchan, E. M.; Sekula, L. K. \& McCrady, C. S. Dimensions of the posterior fossa in patients symptomatic for Chiari I malformation but without cerebellar tonsillar descent. Cerebrospinal Fluid Res., 2:11, 2005.

Smooker, W. R. Craniovertebral junction: normal anatomy, craniometry, and congenital anomalies. Radiographics, 14(2):255-77, 1994.

Snell, R. S. Topografik Klinik Anatomi. Palme Yayıncılık. 9 Baskı. 2015.

Standring, S. Gray's Anatomy. The Anatomical Basis of Clinical Practice. 40th ed. Barcelona, Churchill Livingstone Elsevier, 2008.

Seker, A. \& Rhoton, A. L. The Anatomy of the Posterior Cranial Fossa. In: Özek, M. M.; Cinalli, G.; Maixner, W. \& Sainte-Rose, C. (Eds.). Posterior Fossa Tumors in Children. Amsterdam, Springer, 2015.

Tastemur, Y.; Sabancıgulları, V.; Salk, I .; Sönmez, M. \& Çimen, M. The relationship of the posterior cranial fossa, the cerebrum, and cerebellum morphometry with tonsiller herniation. Iran J. Radiol., 14(1):e24436, 2017.

Tsara, V.; Serasli, E.; Kimiskidis, V.; Papagianopoulos, S.; Katsaridis, V.; Fylaktakis, M.; Christaki, P. \& Kazis, A. Acute respiratory failure and sleep-disordered breathing in Arnold-Chiari malformation. Clin. Neurol. Neurosurg., 107(6):521-4, 2005.

Vurdem, Ü. E.; Acer, N.; Ertekin, T.; Savranlar, A. \& Inci, M. F. Analysis of the volumes of the posterior cranial fossa, cerebellum, and herniated tonsils using the stereological methods in patients with Chiari type I malformation. ScientificWorldJournal, 2012:616934, 2012.

Corresponding author:

Dr. Sema Polat

Cukurova University Faculty of Medicine

Department of Anatomy

Adana

TURKEY

E-mail: sozandac@cu.edu.tr

Received: 23-03-2021

Accepted: 26-06-2021 